\title{
Knockdown of DPP4 promotes the proliferation and the activation of the CREB/aromatase pathway in ovarian granulosa cells
}

\author{
LINA LIN and LIMAN WANG \\ Center for Reproductive Medicine, Shantou Central Hospital, Shantou, Guangdong 515041, P.R. China
}

Received July 9, 2021; Accepted October 15, 2021

DOI: $10.3892 / \mathrm{mmr} .2022 .12589$

\begin{abstract}
Dipeptidyl peptidase 4 (DPP4) has been revealed to be upregulated in women suffering from polycystic ovary syndrome (PCOS), which is a common reproductive disorder. The present study was designed to investigate the effects of inhibition of DPP4 expression on the proliferation of ovarian granulosa cells as well as on the activation of the cAMP response element-binding protein (CREB)/aromatase pathway. The expression levels of DPP4 in rat serum samples with or without PCOS and ovarian granulosa cells (KGN cells) were detected using reverse transcription-quantitative PCR (RT-qPCR) and western blot analyses. Cell viability and cell cycle progression were detected using the Cell Counting Kit- 8 assay and flow cytometric analysis, respectively. The 5-ethynyl-2'-deoxyuridine assay was employed to detect the proliferation of glycolaldehyde-bovine serum albumin (GOA-BSA)-treated KGN cells. In addition, RT-qPCR and western blot analyses were applied to detect the expression levels of CREB, specific cell cycle-associated proteins and cytochrome P450 (CYP) 19A1 and CYP11A1 enzymes in KGN cells. The expression levels of DPP4 were upregulated in rats with PCOS. Inhibition of DPP4 expression promoted the proliferation and cell cycle arrest of KGN cells. It was also revealed that the expression levels of cell cycle-associated proteins were upregulated in DPP4-silenced KGN cells. In addition, their proliferation was decreased following treatment with GOA-BSA, while the addition of sitagliptin partially reversed these effects. Additionally, sitagliptin reversed the inhibitory effects caused by GOA-BSA treatment on the cell cycle progression and on the activation of the CREB/aromatase pathway in KGN cells, as determined by the increased expression levels of the cell cycle-associated proteins as well as those of the CREB protein and the CYP19A1 and CYP11A1
\end{abstract}

Correspondence to: Dr Liman Wang, Center for Reproductive Medicine, Shantou Central Hospital, 114 Waima Road, Shantou, Guangdong 515041, P.R. China

E-mail:wlman2021@126.com

Key words: dipeptidyl peptidase 4, polycystic ovary syndrome, cAMP response element-binding protein/aromatase, advanced glycation end products enzymes. In conclusion, inhibition of DPP4 expression promoted the proliferation of KGN cells and the activation of the CREB/aromatase pathway.

\section{Introduction}

Polycystic ovary syndrome (PCOS) is a heterogeneous and chronic condition featuring hirsutism, anovulation and polycystic ovaries with a prevalence of $10 \%$ (1-3). Patients with PCOS are vulnerable to reproductive abnormalities $(4,5)$, insulin resistance (6), coronary heart disease (7), anxiety and depression (8). In addition, $>30 \%$ of infertility results from PCOS (9). It has been revealed that there are multiple causes of PCOS and these are attributed to genetic and environmental factors, which are prominent contributors to the development of this disease (10). Despite the severity and high incidence rate of PCOS, effective methods for its treatment are limited. Additionally, an insufficient number of research studies have examined its mechanism of development (9). Therefore, a more effective therapeutic treatment for PCOS is required and the investigation of its mechanism of action may contribute to this process.

Dipeptidyl peptidase 4 (DPP4), also known as cluster of differentiation 26, is a type II transmembrane protein released from the cell membrane in a non-classical secretory mechanism. The release of DPP4 may be detected in plasma as well as in different tissues, such as the liver, spleen, lung and ovaries (11). A previous study demonstrated that DPP4 overexpression caused hepatic insulin resistance and promoted liver steatosis, while its inhibition could improve hepatic insulin sensitivity and prevent further accumulation of ectopic fat in the liver (12). Kawasaki et al (13) reported that the inhibition of DPP4 expression could alleviate lipopolysaccharide-induced lung injury in mice and potentially serve as a therapeutic drug for acute respiratory distress syndrome. Furthermore, it was revealed that DPP4 levels were elevated in patients with PCOS and that inhibition of DPP4 expression could be used to treat the development of this condition (14). During the progression of PCOS, estrogen induces cAMP response element-binding protein (CREB) expression in order to promote transcription of cytochrome P450 (CYP) 19A1 and CYP11A1. This in turn reduces the development of the disease (15). However, the effects of the inhibition of DPP4 expression on ovarian granulosa cell proliferation and on the activation of the CREB/aromatase pathway remain unknown. Therefore, the 
present study was designed to explore the role of inhibition of DPP4 expression in ovarian granulosa cell proliferation and on the activation of the CREB/aromatase pathway.

Advanced glycation end products (AGEs) refer to the early glycation products, which undergo further reactions, such as rearrangements and dehydration to become irreversibly cross-linked, fluorescent and senescent macroprotein derivatives (16). By stimulating DPP4 production, AGEs evoke endothelial cell damage (17). AGEs significantly increase DPP4 production, which in turn induces inflammatory reactions in proximal tubular cells (18). Previous studies have also demonstrated that AGEs are closely associated with the pathogenesis of PCOS, as well as its metabolic and reproductive consequences $(19,20)$. In addition, the expression levels of DPP4 are upregulated in the serum and ovarian granulosa cells in patients with PCOS (21). In particular, glycolaldehyde (GOA)-derived AGEs are deemed toxic AGEs, and long term-incubation of GOA with bovine serum albumin (BSA) produces toxic AGEs (22). Therefore, the present study explored whether AGEs could induce the expression of DPP4 in granulosa cells and whether inhibition of DPP4 expression could alleviate the suppressed proliferation of GOA-BSA-treated ovarian granulosa cells.

\section{Materials and methods}

Experimental animals. A total of 20 adult female Sprague Dawley rats, aged 6 weeks, weighing $82 \pm 5 \mathrm{~g}$ were provided by the Animal Center of the Chinese Academy of Sciences and were randomly divided into a control and a PCOS group $(n=10$ per group) (23). Rats were housed in a room with controlled temperature $\left(21-22^{\circ} \mathrm{C}\right)$, and in a 12 -h light/dark cycle, with access to food and water ad libitum. The experiment was conducted in strict accordance to the Guidelines for the Care and Use of Laboratory Animals (24) and the '3R' principle (25). All experiments received approval from the Experimental Animal Ethics Committee of Zhaofenghua Biological Technology Co., Ltd. (approval number IACUC-20201012-16).

Establishment of a PCOS animal model. In the PCOS group $(\mathrm{n}=10)$, the rats were intragastrically administered $0.5 \mathrm{mg} / \mathrm{kg}$ letrozole (Jiangsu Hengrui Medicine Co., Ltd.) dissolved in $1 \%(\mathrm{w} / \mathrm{w})$ carboxymethylcellulose (CMC; Beyotime Institute of Biotechnology) and fed with a high fat diet for 21 days (26). The control group of 10 rats received vehicle only (1\% aqueous solution of CMC) once daily (p.o.). To obtain vaginal smears, rats were restrained by hand, a sterile cotton swab moistened with normal saline was inserted into the vagina of the animal to dip in the exfoliated cells via gentle rotation in the vagina (27-29). The whole movement was gentle to ensure that the rats experienced no pain. The vaginal smears of the rats were monitored 1 week following modeling and the estrous cycle was used to assess the successful establishment of the model. The animal health and behavior were monitored every day, and the following humane endpoints were used in the present study: Rapid loss of $15 \%$ of the original body weight; continuous inability of the rats to gain weight; and inability to eat and drink on their own. On the 30th day, the rats were anesthetized with $2 \%$ sodium pentobarbital (intraperitoneal injection, $40 \mathrm{mg} / \mathrm{kg}$ ) and then the blood samples $(4-6 \mathrm{ml})$ from the abdominal aorta were collected, following cervical dislocation of the spine. When the cessation of the heartbeat and breathing of the rats was verified, and there were no reflexes, the death of the rats was confirmed. The blood samples were centrifuged at $1,000 \mathrm{x} \mathrm{g} / \mathrm{min}$ for $10 \mathrm{~min}$ at $4^{\circ} \mathrm{C}$ to obtain the serum samples. The granular cells from the ovaries of control and PCOS rats were isolated as previously reported (30).

Hematoxylin and eosin $(H \& E)$ staining. The ovarian tissues of control and PCOS rats were collected and fixed with $10 \%$ formaldehyde at room temperature. Following dehydration, tissues were embedded in paraffin and sliced into pieces (cross-sections, $5 \mu \mathrm{m}$ ). After being deparaffinized and rehydrated, an H\&E staining kit (product code ab245880; Abcam) was applied to the sections following the manufacturer's protocol, and the sections were observed by light microscope, Leica DM3000 (Leica Microsystems Ltd.; magnification, x200).

Cell culture, treatment and transfection. The immortalized human granulosa-like tumor cell line, KGN, which maintained the physiological characteristics of ovarian cells and has been widely used to study PCOS (30-33) was purchased from the American Type Culture Collection (ATCC). The cells were cultured in DMEM (Gibco; Thermo Fisher Scientific, Inc.) supplemented with $10 \%$ fetal bovine serum (FBS; Gibco; Thermo Fisher Scientific, Inc.), $100 \mathrm{U} / \mathrm{ml}$ penicillin and $100 \mu \mathrm{g} / \mathrm{ml}$ streptomycin (Invitrogen; Thermo Fisher Scientific, Inc.) at $37^{\circ} \mathrm{C}$ in a humidified atmosphere with $5 \% \mathrm{CO}_{2}$.

In order to knockdown DPP4 expression in $\mathrm{KGN}$ cells, small interfering RNA (siRNA) targeting DPP4 (siRNA-DPP4-1 and siRNA-DPP4-2) and the corresponding negative control (siRNA-NC) were synthesized by GenScript. The transfection of $2 \mu \mathrm{g} / \mathrm{ml}$ vectors into $\mathrm{KGN}$ cells at $37^{\circ} \mathrm{C}$ for $10 \mathrm{~h}$ was conducted using Lipofectamine ${ }^{\circledR} 2000$ transfection reagent (Invitrogen; Thermo Fisher Scientific, Inc.) as determined by the manufacturer's protocol. At $48 \mathrm{~h}$ post-transfection, cells were used for subsequent experiments. The sequence for siRNAs were as follows: siRNA-DPP4-1 forward, 5'-GUA CCUCCUUAUUCAUGGATT-3' and reverse, 5'-UCCAUG AAUAAGGAGGUACTT-3'; siRNA-DPP4-2 forward, 5'-CAC CGUGGAAGGUUCUUCUTT-3' and reverse, 5'-AGAAGA ACCUUCCACGGUGTT-3'; siRNA-NC, forward, 5'-UUC UCCGAACGUGUCACGUTT-3' and reverse, 5'-TTAAGA GGCUUGCACAGUGCA-3'.

GOA-BSA was prepared as previously reported (22), and KGN cells were treated with $400 \mu \mathrm{g} / \mathrm{ml}$ GOA-BSA for 24 , 48, 72 and $96 \mathrm{~h}$ with or without sitagliptin (100 nM; APeXBIO Technology LLC) co-treatment for $24 \mathrm{~h}$.

Reverse transcription-quantitative PCR (RT-qPCR). Total RNA from cells or samples was extracted using TRlzol ${ }^{\circledR}$ reagent (Thermo Fisher Scientific, Inc.). The samples were reverse transcribed into cDNA using a RevertAid First Strand cDNA Synthesis kit (cat. no. K1622; Invitrogen; Thermo Fisher Scientific, Inc.) according to the manufacturer's protocol. qPCR was performed using a SYBR-Green kit (QuantiNova SYBR Green RT-PCR kit, cat. no. 208152; Qiagen AB). The thermocycling conditions were: Initial denaturation at $95^{\circ} \mathrm{C}$ for $8 \mathrm{~min}$; denaturation at $95^{\circ} \mathrm{C}$ for $25 \mathrm{sec}$, annealing at $60^{\circ} \mathrm{C}$ for $1 \mathrm{~min}$ (37 cycles); and final extension at $72^{\circ} \mathrm{C}$ for $10 \mathrm{~min}$. GAPDH was used as an endogenous control and the calculation of the relative 
gene expression was performed using the $2^{-\Delta \Delta \mathrm{Cq}}$ method (34). Primers are as follows: DPP4 (rat) forward, 5'-ATTCCGTAC CCAAAGGCAGG-3' and reverse, 5'-AGGCCACGTCAC ACAAGTAG-3'; DPP4 (human) forward, 5'-TCTGCTGAA CAAAGGCAATGA-3' and reverse, 5'-CTGTTCTCCAAG AAAACTGAGC-3'; CREB forward, 5'-TAGTGCCCAGCA ACCAAGT-3' and reverse, 5'-ACATGTTACCATCTTCAA ACTGAC-3'; CYP19A1, forward, 5'-ACTTATCCTATCAGG ACGGAAGGT-3' and reverse, 5'-AGGGGGCAATTTAGA GTCGC-3'; CYP11A1 forward, 5'-GCTGAAGTGGAGCAG GTACA-3' and reverse, 5'-CTTTGACCAGGACTGAGCGT-3'; GAPDH (rat) forward, 5'-GCATCTTCTTGTGCAGTGCC-3' and reverse, 5'-TACGGCCAAATCCGTTCACA-3'; GAPDH (human) forward, 5'-AATGGGCAGCCGTTAGGAAA-3' and reverse, 5'-GCGCCCAATACGACCAAATC-3'.

Western blot analysis. Total proteins from cells or samples were extracted using RIPA lysis buffer (Beijing Solarbio Science \& Technology Co., Ltd.). A BCA kit (Beyotime Institute of Biotechnology) was used to quantify the protein concentration. Subsequently, the samples (30 $\mu \mathrm{g}$ per lane) were separated with $12 \%$ SDS-PAGE and transferred to PVDF membranes (MilliporeSigma). Following blocking with 5\% non-fat milk for $2 \mathrm{~h}$ at room temperature, the membranes were incubated with the corresponding primary antibodies at $4{ }^{\circ} \mathrm{C}$ overnight. Subsequently, a HRP-conjugated goat anti-rabbit secondary antibody (1:10,000; cat. no. ab205718; Abcam) was used to incubate the membranes for $2 \mathrm{~h}$ at room temperature. Finally, an ECL kit (Beyotime Institute of Biotechnology) was used to determine the relative protein levels. The rabbit primary antibodies (Abcam) included: DPP4 (1:2,000; cat. no. ab187048), cyclin-dependent kinases (CDKs) CDK2 (1:2,000; cat. no. ab32147), CDK4 (1:2,000; cat. no. ab108357), CDK6 (1:2,000; cat. no. ab124821), cell cycle proteins (cyclins) cyclin B (1:2,000; cat. no. ab32053), cyclin E (1:2,000; cat. no. ab33911), CREB (1:2,000; cat. no. ab32515), CYP19A1 (1:1,000; cat. no. ab106168), CYP11A1 (1:1,000; cat. no. ab272494), GAPDH (1:2,500; cat. no. ab9485) and $\beta$-actin (1:2,000; cat. no. ab8227). The densitometry analysis was performed using ImageJ software (version 1.8.0; National Institutes of Health).

Cell Counting Kit-8 (CCK-8) assay. The effects of silencing DPP4 expression were investigated on KGN cell viability using the CCK- 8 assay. The cells (2,000 cells per well) were incubated in 96-well plates for $24,48,72$ and $96 \mathrm{~h}$ at $37^{\circ} \mathrm{C}$. Subsequently, $10 \%$ CCK-8 solution (Beyotime Institute of Biotechnology) was added to each well and the cells were incubated for an additional $2 \mathrm{~h}$ at $37^{\circ} \mathrm{C}$. Subsequently, the absorbance was determined at $450 \mathrm{~nm}$ using a microplate reader (Thermo Fisher Scientific Inc.).

5-Ethynyl-2'-deoxyuridine (EdU) staining. The transfected KGN cells were seeded into 96-well plates and incubated with EdU solution (Beyotime Institute of Biotechnology) for $4 \mathrm{~h}$ at room temperature. Following fixation with $4 \%$ paraformaldehyde at room temperature for $1 \mathrm{~h}$, the cells were stained with 1X Apollo567 solution for $30 \mathrm{~min}$ at room temperature in the dark and DAPI solution was used to stain the cell nuclei at room temperature for $5 \mathrm{~min}$. A fluorescent microscope was employed to observe the results (magnification, $\mathrm{x} 200$ ) and
Image J software (version 1.8.0; National Institutes of Health) was used to analyze the percentage of EdU-positive nuclei (green) compared with the total percentage of the nuclei (stained with blue fluorescence), and then the data was revealed as relative to the control group (normal KGN cells).

Flow cytometry. The transfected KGN cells were centrifuged at $1,000 \mathrm{x} \mathrm{g} / \mathrm{min}$ for $30 \mathrm{sec}$ at $4^{\circ} \mathrm{C}$ and the supernatant was discarded. Subsequently, $1 \mathrm{ml}$ PBS was used to resuspend the cells. The precipitated cells were resuspended using $100 \mu \mathrm{l}$ RNase A at $37^{\circ} \mathrm{C}$ for $30 \mathrm{~min}$. A total of $400 \mu \mathrm{l}$ PI staining solution was added and the cells were stained in the dark at room temperature for $10 \mathrm{~min}$. Finally, flow cytometry (BD FACS; BD Biosciences) was used to assess the cell cycle and the data was analyzed by FlowJo version 7.6 (FlowJo LLC).

Statistical analysis. All data are presented as the mean \pm SD of three independent experiments after normalization to the control group, and statistical analysis was performed using SPSS 20.0 (IBM Corp.). Statistical differences between two groups were determined using an unpaired Student's t-test, while one-way ANOVA was carried out with Tukey's multiple comparison post-hoc test. $\mathrm{P}<0.05$ was considered to indicate a statistically significant difference.

\section{Results}

DPP4 expression is increased in serum samples of rats with PCOS and KGN cells. To validate the success of the PCOS animal model, ovarian tissues from control and PCOS rats were observed by H\&E staining. As illustrated in Fig. 1A, the structure of ovarian tissues in the control rats was well arranged, while in the PCOS rats it was arranged in disorder. In addition, control rats exhibited ovarian follicles at a variety of stages, whereas ovarian cystic expansion and a significant drop in the number of granular cells was observed in the ovaries of PCOS rats. The aforementioned results indicated the successful establishment of the PCOS animal model. Subsequently, the relative mRNA expression levels of DPP4 were determined in the serum samples of rats with PCOS using RT-qPCR analysis. DPP4 expression was significantly increased in rats with PCOS ( 3.4-fold of the control) compared with that of the control group (Fig. 1B). In addition, DPP4 expression in granular cells from the ovaries of control and PCOS rats was detected using RT-qPCR and western blot analyses. The relative expression levels of DPP4 were significantly increased in granular cells from the ovaries of PCOS rats compared with those of the control rats (Fig. 1C). The aforementioned results suggested that DPP4 exhibited high expression in the PCOS group.

Knockdown of DPP4 expression promotes the proliferation of KGN cells. The effects of silencing DPP4 expression were investigated on KGN cell proliferation. KGN cells were transfected with siRNA-DPP4 and the relative mRNA and protein expression levels were detected using RT-qPCR and western blot analyses, respectively. The expression levels of DPP4 were significantly decreased compared with those noted in the siRNA-NC group, whereas siRNA-DPP4-1 contributed to the lowest DPP4 expression (0.3 vs. 0.36; Fig. $1 \mathrm{D}$ and E). 

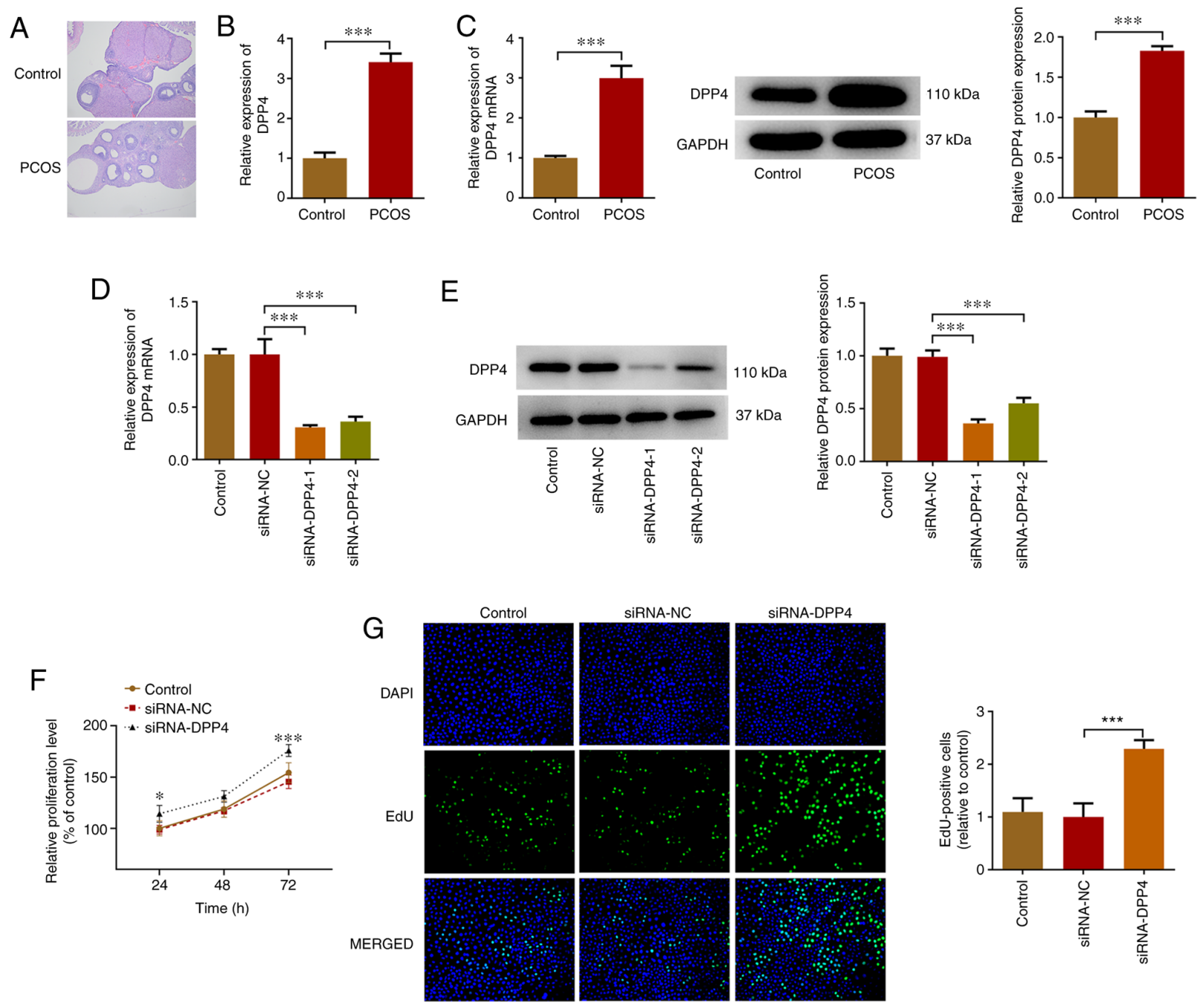

Figure 1. Expression levels of DPP4 are increased in the serum of rats with PCOS and KGN cells, while inhibition of DPP4 expression promotes the proliferation of KGN cells. (A) Ovarian tissues from control and PCOS rats were subjected to H\&E staining (magnification, x200). (B) RT-qPCR was used to assess the relative mRNA expression levels of DPP4 in the serum of rats with PCOS. (C) DPP4 RNA and protein expression levels in granular cells from the ovaries of control and PCOS rats were determined using RT-qPCR and western blot analyses. The relative mRNA and protein expression levels of DPP4 in KGN cells that were transfected with indicated vectors were detected using (D) RT-qPCR and (E) western blot analyses, respectively. The proliferation of DPP4-silenced KGN cells was detected using (F) Cell Counting Kit-8 and (G) 5-ethynyl-2'-deoxyuridine staining assays. ${ }^{*} \mathrm{P}<0.05,{ }^{* * *} \mathrm{P}<0.001$. DPP4, dipeptidyl peptidase 4 ; PCOS, polycystic ovary syndrome; RT-qPCR, reverse transcription-quantitative PCR; EdU, 5-ethynyl-2'-deoxyuridine; siRNA, small interfering RNA; NC, negative control.

Therefore, siRNA-DPP4-1 was selected for subsequent experiments. In addition, the proliferation of DPP4-silenced KGN cells was detected using the CCK-8 and EdU staining assays. The results demonstrated that the proliferation of KGN cells was significantly increased due to DPP4 silencing in comparison with that of the siRNA-NC group (Fig. 1F and G).

Knockdown of DPP4 expression accelerates the cell cycle of $K G N$ cells. Flow cytometry was employed to assess the role of the inhibition of DPP4 expression on the cell cycle of KGN cells. The cell cycle is divided into the $G_{1}, S, G_{2}$ and $M$ phases and each phase exhibits different characteristics (23). Different functions are required for the transition from one phase to the next (35). The number of DPP4-silenced KGN cells at the $\mathrm{G}_{1}$ and $\mathrm{G}_{2} / \mathrm{M}$ phases was slightly diminished compared with that of the siRNA-NC group, whereas the number of cells at the $\mathrm{S}$ phase was significantly increased by DPP4 silencing in comparison with that of siRNA-NC (Fig. 2A and B). The cell cycle is regulated via the activation of cell cycle proteins (cyclins) and CDKs $(36,37)$. Therefore, the expression levels of CDK2, CDK4, CDK6, cyclin D1, cyclin E and cyclin B were detected using western blot analysis. The results indicated that the expression levels of the cell cycle proteins (cyclin D1, cyclin E and cyclin B) and CDKs (CDK2, CDK4, CDK6) were upregulated in the DPP4-silenced KGN cells compared with those of the siRNA-NC group (Fig. 2C). The aforementioned results revealed that silencing of DPP4 expression could effectively accelerate the cell cycle of KGN cells.

Knockdown of DPP4 expression induces upregulation of the expression levels of CREB, CYP19A1 and CYP11A1. Subsequently, the expression levels of CREB, CYP19A1 and CYP11A1 were detected via RT-qPCR and western blot analyses. The transcription factor CREB and the enzymes CYP19A1 and CYP11A1 had higher expression levels in DPP4-silenced KGN cells compared with those of the siRNA-NC group, revealing that DPP4 silencing induced the activation of CREB and the transcription of aromatase (Fig. 3). 

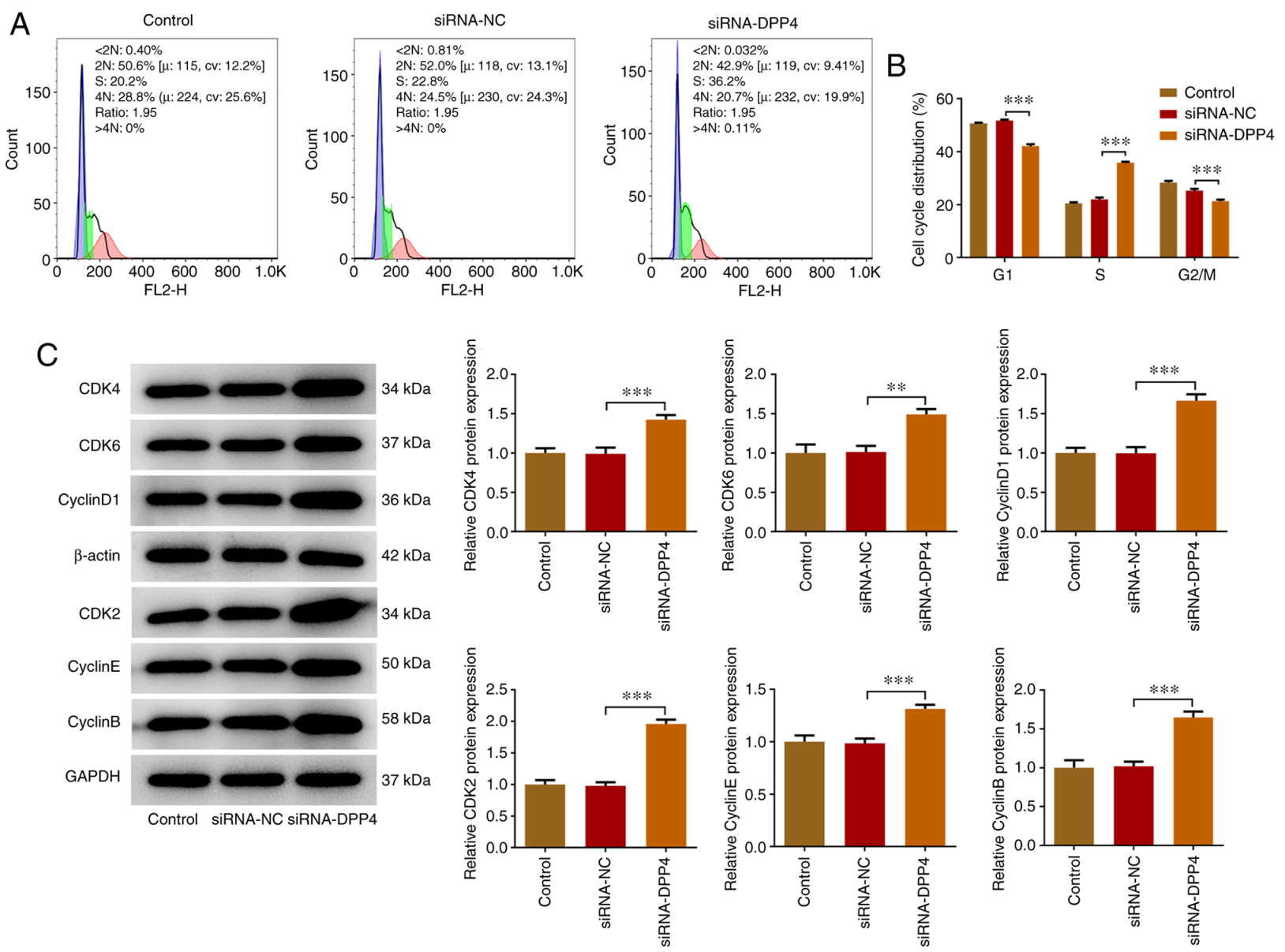

Figure 2. Knockdown of DPP4 expression accelerates the cell cycle of KGN cells. (A and B) Flow cytometry was employed to investigate the role of DPP4 knockdown on the cell cycle distribution including $\mathrm{G}_{1}, \mathrm{~S}$ and $\mathrm{G}_{2} / \mathrm{M}$ of KGN cells. (C) The expression levels of CDK2, CDK4, CDK6, cyclin D1, cyclin E and cyclin B were detected using western blot analyses. ${ }^{* *} \mathrm{P}<0.01$ and ${ }^{* * *} \mathrm{P}<0.001$. DPP4, dipeptidyl peptidase 4; CDK, cyclin-dependent kinase; siRNA, small interfering RNA; NC, negative control.
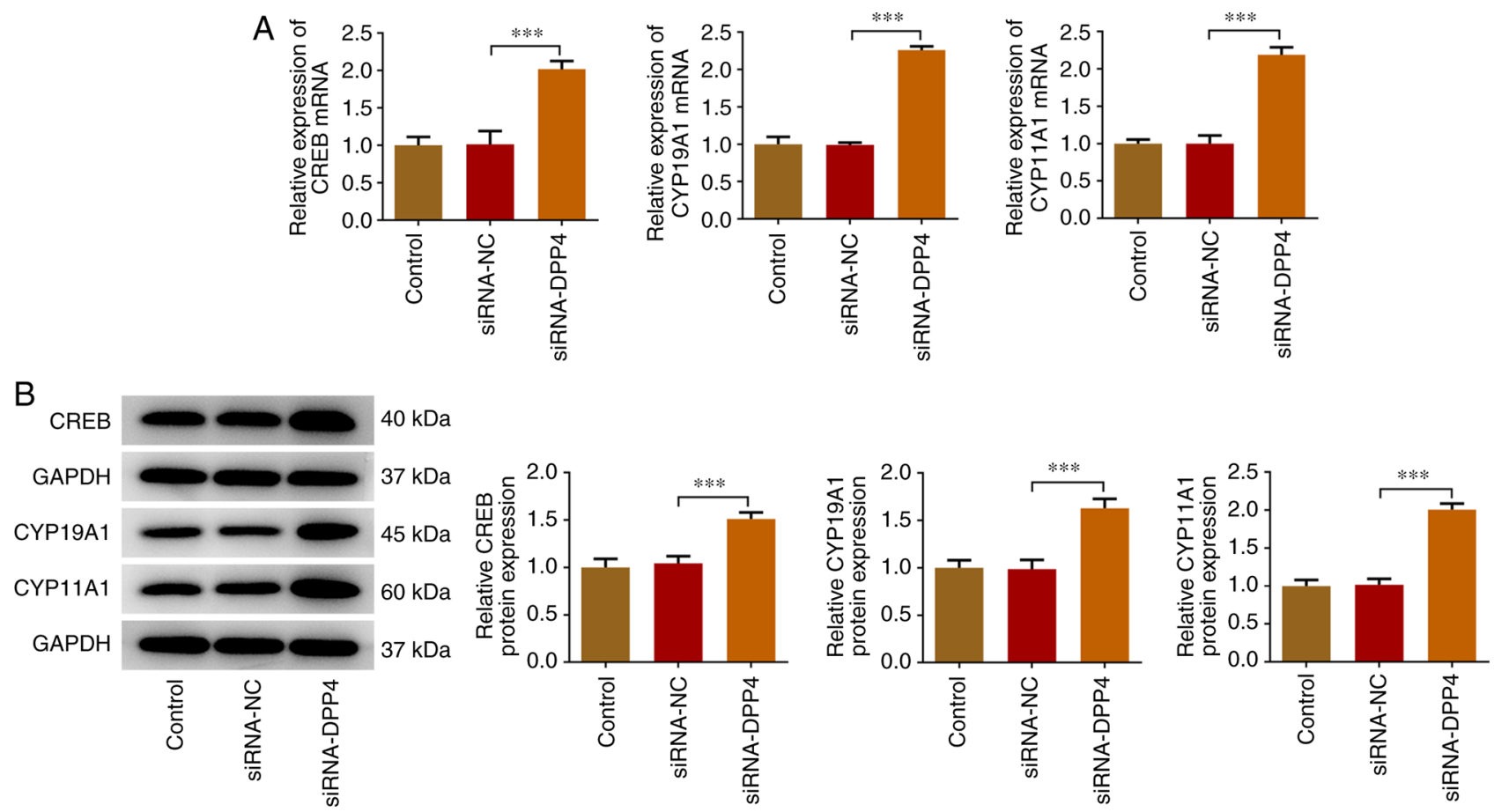

Figure 3. Knockdown of DPP4 expression upregulates the expression levels of CREB, CYP19A1 and CYP11A1. The expression levels of CREB, CYP19A1 and CYP11A1 were assessed using (A) reverse transcription-quantitative PCR and (B) western blot analysis. ${ }^{* * *}$ P $<0.001$. DPP4, dipeptidyl peptidase 4; CREB, cAMP response element-binding protein; CYP, cytochrome P450; siRNA, small interfering RNA; NC, negative control. 
A

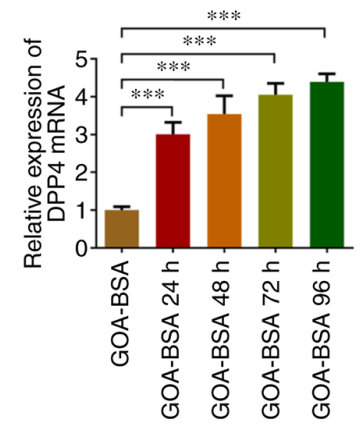

B

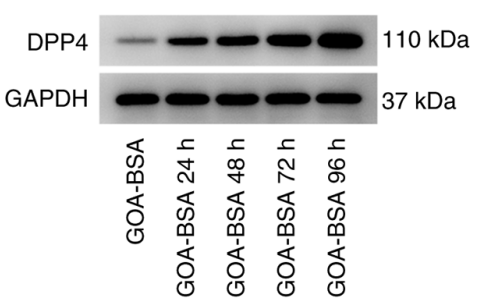

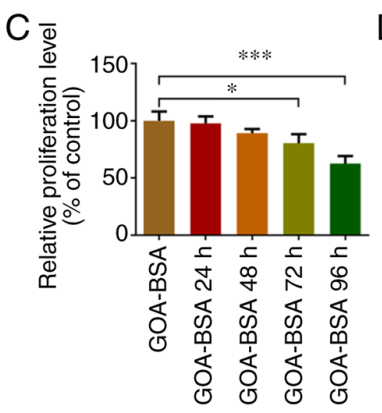

$\mathrm{F}$

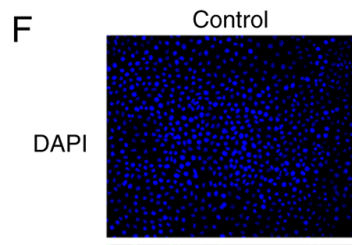

$\mathrm{D}$ -

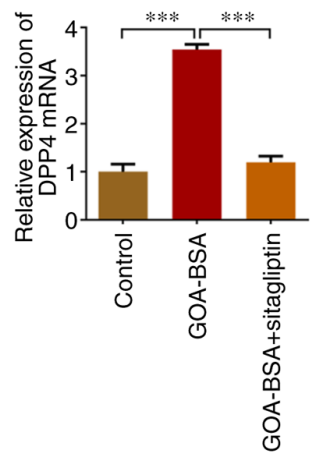

E
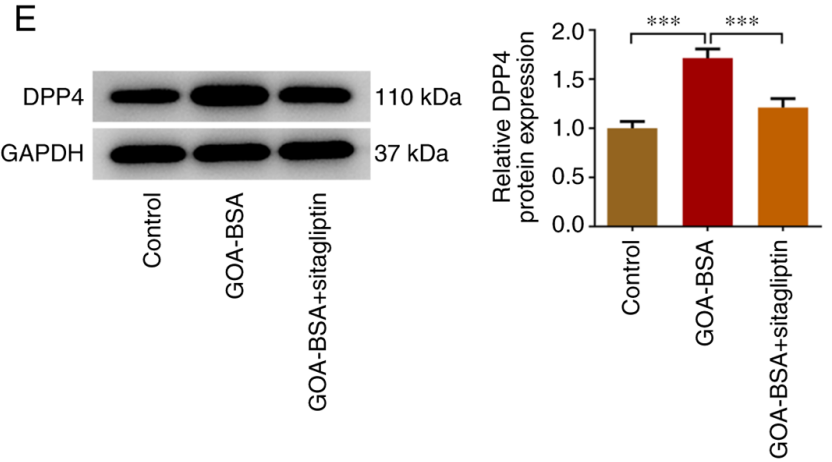
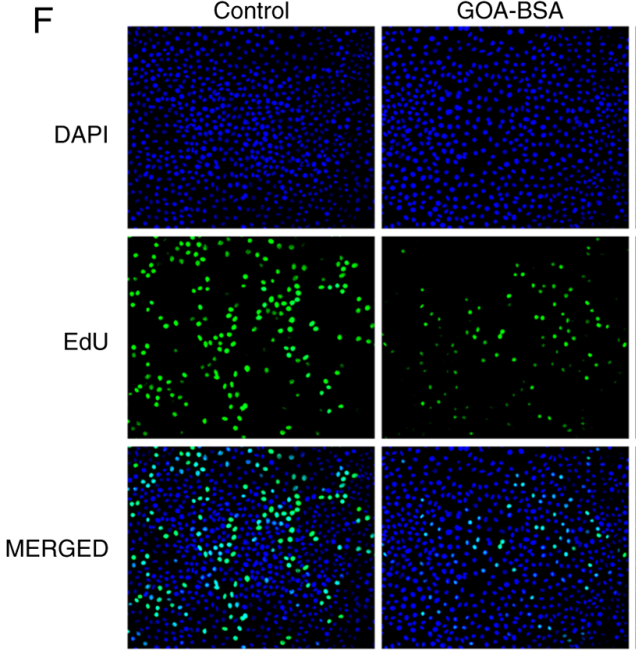

GOA-BSA+sitagliptin
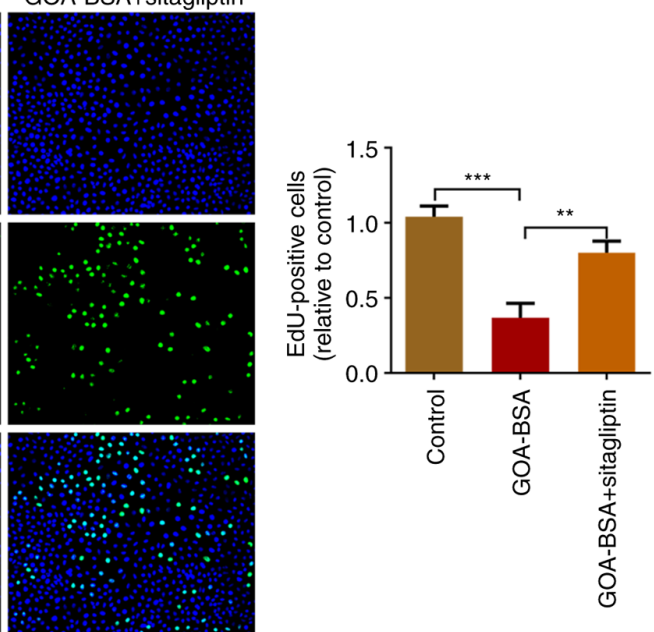

Figure 4. Expression levels of DPP4 are upregulated in KGN cells after GOA-BSA treatment, while sitagliptin reverses the inhibitory effects of GOA-BSA treatment on KGN cell proliferation. The expression levels of DPP4 in GOA-BSA-treated KGN cells were detected using (A) RT-qPCR and (B) western blot analyses. (C) The proliferation of KGN cells was detected using the Cell Counting Kit-8 assay. The expression levels of DPP4 in GOA-BSA-treated KGN cells were detected using (D) RT-qPCR and (E) western blot analyses. (F) The cell proliferation of KGN cells was detected using the 5-ethynyl-2'-deoxyuridine staining assays. ${ }^{*} \mathrm{P}<0.05,{ }^{* *} \mathrm{P}<0.01$ and ${ }^{* * *} \mathrm{P}<0.001$. DPP4, dipeptidyl peptidase 4; GOA-BSA, glycolaldehyde-bovine serum albumin; RT-qPCR, reverse transcription-quantitative PCR; EdU, 5-ethynyl-2'-deoxyuridine.

GOA-BSA upregulates DPP4 expression and inhibits proliferation of KGN cells, whereas sitagliptin reverses these effects. Previous studies have demonstrated that AGEs are closely associated with the pathogenesis of PCOS as well as its reproductive consequences $(18,19)$. Additionally, DPP4 expression was increased in KGN cells and in serum samples derived from rats with PCOS $(19,20)$. Toxic AGEs were prepared by long term-incubation of GOA with BSA (22). Therefore, GOA-BSA was used to treat KGN cells. DPP4 expression was increased following treatment of the cells with GOA-BSA and this process occurred in a time-dependent manner (Fig. 4A and B). The CCK-8 assay indicated that treatment of KGN cells with GOA-BSA decreased their proliferation in a time-dependent manner (Fig. 4C). Subsequently, $96 \mathrm{~h}$ was selected as the action time of GOA-BSA considering that GOA-BSA treatment for $96 \mathrm{~h}$ resulted in the largest effect (Fig. 4A-C). Increased DPP4 expression was decreased following the addition of sitagliptin, which is an inhibitor of DPP4 (Fig. 4D and E). In addition, the proliferation of KGN cells in GOA-BSA group was increased following concomitant treatment of the cells with GOA-BSA and stimulation by sitagliptin, suggesting that the latter partly abolished the inhibitory effects of GOA-BSA on KGN cells (Fig. 4F).

Suppression of DPP4 expression alleviates the inhibitory effects of GOA-BSA on the cell cycle of KGN cells. The 

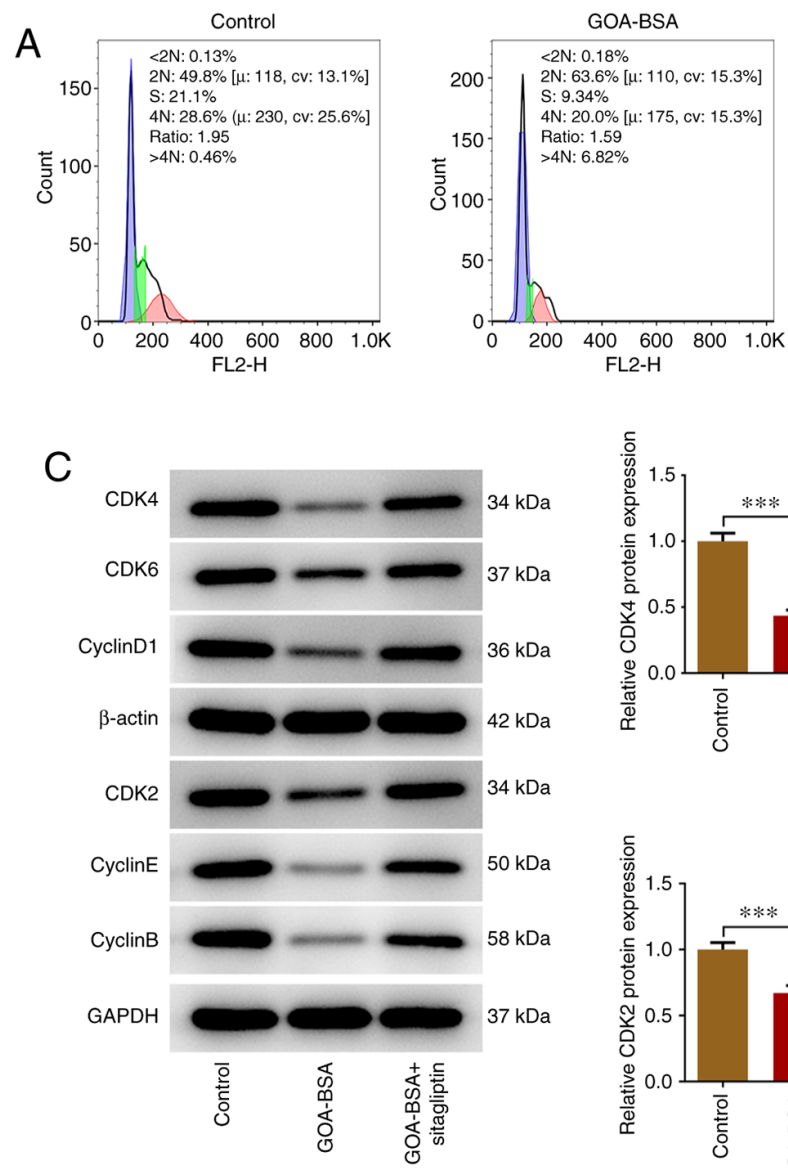
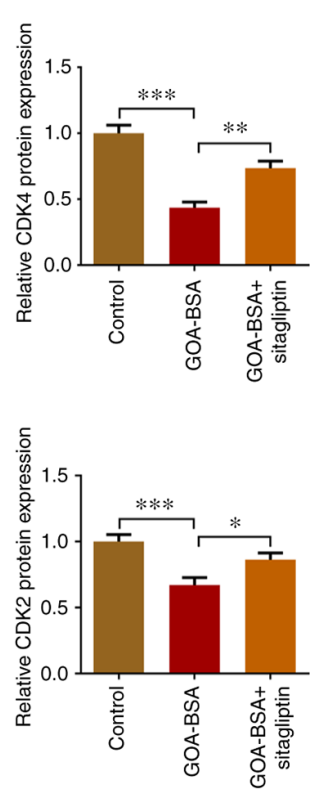
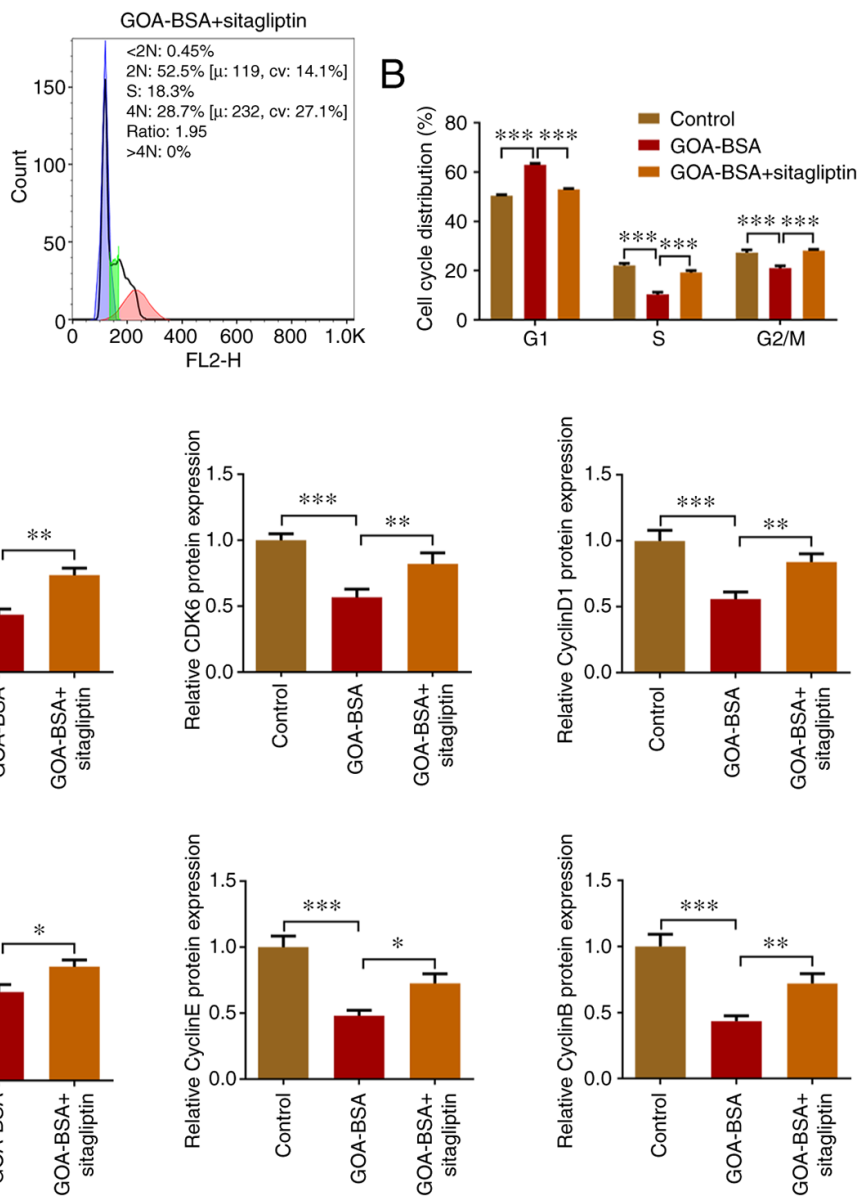

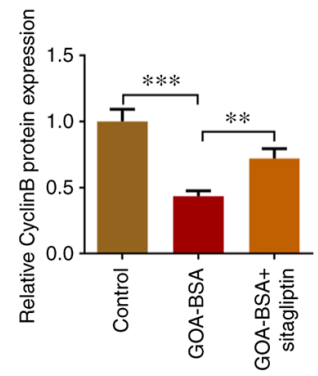

Figure 5. Suppression of DPP4 expression alleviates the inhibitory effects of GOA-BSA treatment on the cell cycle of KGN cells. (A and B) Flow cytometry was employed to assess the role of inhibition of DPP4 expression in the cell cycle distribution including $\mathrm{G}_{1}, \mathrm{~S}$ and $\mathrm{G}_{2} / \mathrm{M}_{\mathrm{N}}$ of $\mathrm{KGN}$ cells. (C) The expression levels of CDK2, CDK4, CDK6, cyclin D1, cyclin E and cyclin B were detected using western blot analyses. ${ }^{*} \mathrm{P}<0.05$, ${ }^{* *} \mathrm{P}<0.01$ and ${ }^{* * *} \mathrm{P}<0.001 . \mathrm{DPP} 4, \mathrm{dipeptidyl}$ peptidase 4; CDK, cyclin-dependent kinase; GOA-BSA, glycolaldehyde-bovine serum albumin.

number of cells in the $\mathrm{S}$ and $\mathrm{G}_{2} / \mathrm{M}$ phases was decreased following their treatment with GOA-BSA, while the addition of sitagliptin alleviated the inhibitory effects of GOA-BSA (Fig. 5A and B). In contrast to these observations, the increased number of cells at the $\mathrm{G}_{1}$ phase was diminished by sitagliptin treatment. In addition, sitagliptin promoted the effects on the cell cycle-associated proteins, as demonstrated by the increased expression levels of CDK4, CDK6, cyclin D1, CDK2, cyclin $\mathrm{E}$ and cyclin B compared with those noted in the GOA-BSA group (Fig. 5C).

Suppression of DPP4 expression upregulates the expression levels of CREB, CYP19A1 and CYP11A1 in GOA-BSA-treated $K G N$ cells. The mRNA and protein expression levels of CREB, CYP19A1 and CYP11A1 were significantly decreased due to GOA-BSA treatment compared with those noted in the control group. However, the addition of sitagliptin partially increased the expression levels of CREB, CYP19A1 and CYP11A1, indicating that it could abolish the inhibitory effects of GOA-BSA on KGN cells (Fig. 6A and B).

\section{Discussion}

PCOS is a common endocrinopathy affecting a large part of the global population. It contributes to ovulatory infertility in women, accounting for $75 \%$ of non-ovulatory infertile women of childbearing age $(38,39)$. It has been reported that one in 5-6 women has severe infertility and irregular menstrual cycles (40). In recent years, PCOS has been a research hotspot and numerous studies have been conducted to examine its mechanism of action (6-10). However, a limited number of studies have investigated the role of DPP4 in the development of PCOS. Therefore, the aim of the present study was to detect DPP4 expression and explore its role in PCOS. First, a PCOS model was established in rats via intragastrical administration of letrozole (26). PCOS is commonly manifested as ovulatory dysfunction, clinical and biochemical excess of androgen levels, and polycystic ovaries (41). In the present study, disarranged structure of ovarian tissues, ovarian cystic expansion and a significant drop in the number of granular cells were observed in the ovary of PCOS rats, suggesting the successful establishment of the PCOS animal model. Subsequently, DPP4 expression was detected in serum samples derived from rats with PCOS and ovarian granulosa cells, indicating that the expression levels of DPP4 were significantly upregulated in this model. To further assess the effects of DPP4 on PCOS, KGN cells were transfected with siRNA-DPP4 and the data indicated that knockdown of DPP4 expression promoted the proliferation and cell cycle progression of KGN cells and the induction of CREB, CYP19A1 and CYP11A1 expression. 

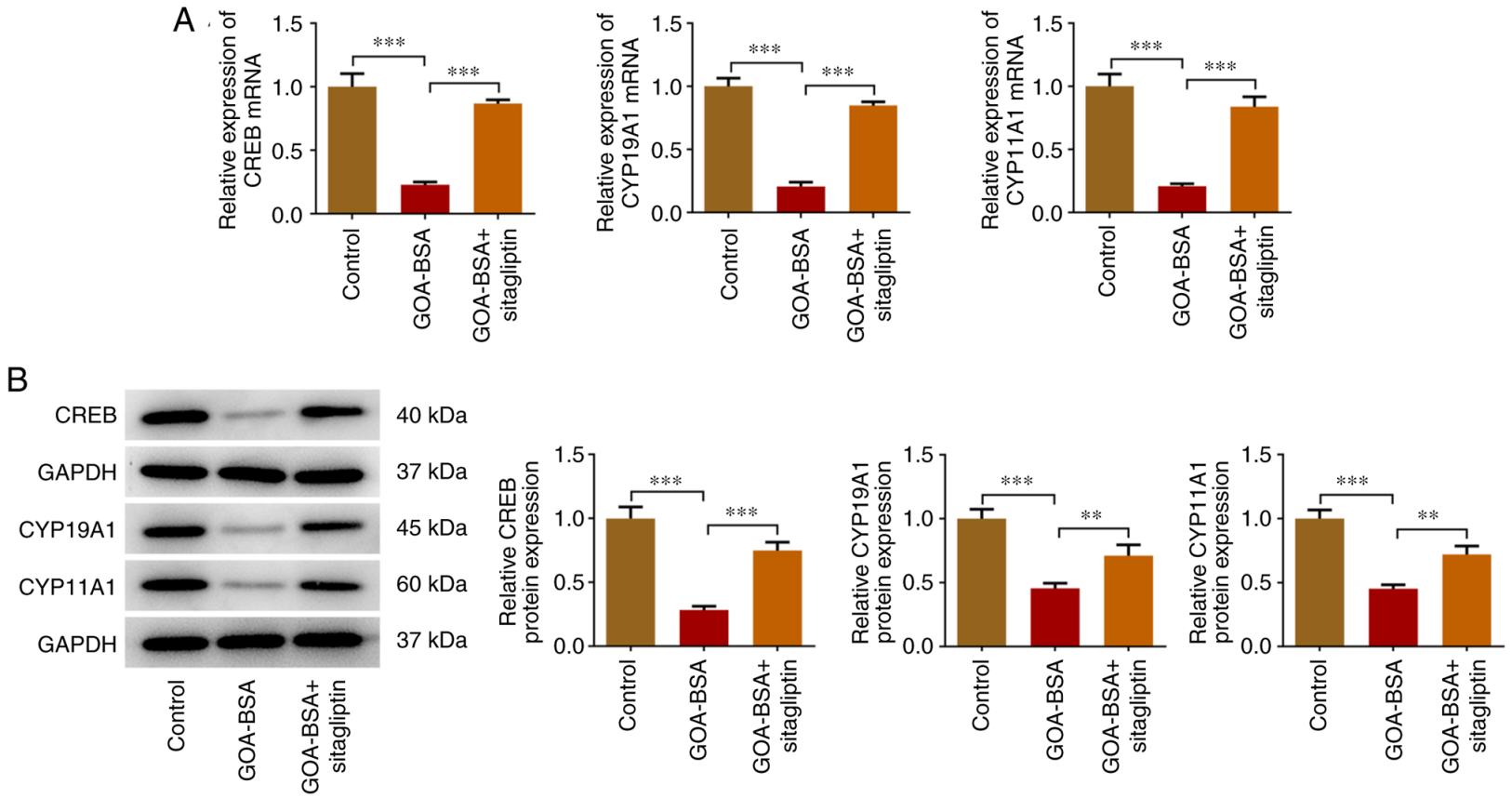

Figure 6. Suppression of DPP4 expression upregulates the expression levels of CREB, CYP19A1 and CYP11A1 in GOA-BSA-treated KGN cells. The expression levels of CREB, CYP19A1 and CYP11A1 were assessed using (A) reverse transcription-quantitative PCR and (B) western blot analyses. ${ }^{* *} \mathrm{P}<0.01$ and ${ }^{* * *} \mathrm{P}<0.001$. DPP4, dipeptidyl peptidase 4; CREB, cAMP response element-binding protein; CYP, cytochrome P450; GOA-BSA, glycolaldehyde-bovine serum albumin.

DPP4 is a ubiquitous multifunctional type II transmembrane protease and a member of the S9 protease family $(29,30)$. It was initially identified in $1963(42,43)$. DPP4 has been revealed to participate in several biological processes, such as inflammation and tumor immunity $(44,45)$. The mRNA and protein expression levels of DPP4 have notably been revealed to be increased in the ovaries (43). Jensterle et al (46) reported that DPP4 expression was increased in PCOS and that treatment of the cells with DPP4 inhibitors, such as alogliptin and pioglitazone, could be a therapeutic therapy for the treatment of this condition. In addition, DPP4 expression was revealed to be upregulated in rats with PCOS (47). In the present study, RT-qPCR and western blot analyses indicated that DPP4 expression was significantly increased in ovarian granulosa cells, which was consistent with the results reported in the aforementioned studies.

AGEs, also known as 'glycotoxins', are the products of non-enzymatic glycation and oxidation of proteins and lipids that are formed during endogenous and exogenous reactions (48-50). A previous study demonstrated that AGEs were an important player in aging, diabetes, atherosclerosis, female fertility and the pathogenesis of cancer (48). Azhary et al (51) reported that AGEs were accumulated in granulosa cells from patients with PCOS. AGEs were also revealed to be associated with the pathogenesis of PCOS $(52,53)$. In addition, it has been demonstrated that induction of AGEs stimulates the release of DPP4 from endothelial cells (54). In the present study, it was revealed that AGEs (GOA-BSA) increased the expression levels of DPP4 and inhibited the proliferation of KGN cells in a time-dependent manner.

Sitagliptin is an effective selective inhibitor of DPP4 specifically designed to inhibit the DPP4 enzyme (55). Ferjan et al (56) demonstrated that sitagliptin was an optimal therapeutic strategy for the treatment of PCOS. Furthermore, a recent study provided encouraging data on a beneficial effect of sitagliptin in reproduction, demonstrating that sitagliptin could improve the maturation of oocytes and quality of embryos in PCOS patients undergoing intracytoplasmic sperm injection (57). In the present study, increased expression of DPP4 caused by treatment of the cells with GOA-BSA was decreased following application of sitagliptin. The proliferation of GOA-BSA-treated KGN cells was diminished, while the addition of sitagliptin partially reversed the effects of GOA-BSA on KGN cells. In addition, sitagliptin alleviated the cell cycle of GOA-BSA-treated KGN cells, while upregulating the expression levels of the cell cycle-associated proteins. Furthermore, sitagliptin promoted the transcription of aromatase in GOA-BSA-induced KGN cells, as demonstrated by the increased expression levels of CREB, CYP19A1 and CYP11A1. However, the present study contains certain limitations, such as the verification of the dose and safety of sitagliptin along with the repeated experiments using rat primary granular cells and the PCOS rat model. Therefore, further studies are required to confirm and expand these conclusions. In addition, to improve the reliability of these preliminary results, further studies may be performed to evaluate other aspects such as apoptosis, autophagy and DNA methylation, in addition to the vitality/proliferation/cell cycle in DPP4-silenced KGN cells.

In summary, the present study indicated that the expression levels of DPP4 were upregulated in rats with PCOS. Treatment of KGN cells with GOA-BSA decreased cell proliferation, the expression levels of the cell cycle-associated proteins and the transcription of aromatase. These effects were reversed following treatment of the cells with sitagliptin. The present study, to the best of our knowledge, for the first time demonstrated the protective effect of DPP4 inhibitor, sitagliptin, against PCOS, providing evidence for the potential value of sitagliptin in serving as an ideal therapy for the treatment of PCOS. 


\section{Acknowledgements}

Not applicable.

\section{Funding}

No funding was received.

\section{Availability of data and materials}

All data generated or analyzed during the present study are included in this published article.

\section{Authors' contributions}

LW contributed to study conception and design. LL and LW contributed to acquisition, analysis and interpretation of data. LL drafted the initial manuscript and LW revised it critically for important intellectual content. LL and LW confirm the authenticity of all the raw data. All authors read and approved the final manuscript.

\section{Ethics approval and consent to participate}

The experiments were conducted in strict accordance with the Guidelines for the Care and Use of Laboratory Animals and the '3R' principle. All experiments received approval from the Experimental Animal Ethics Committee of Zhaofenghua Biological Technology Co., Ltd. (Nanjing, China; approval no. IACUC-20201012-16).

\section{Patient consent for publication}

Not applicable.

\section{Competing interests}

The authors declare that they have no competing interests.

\section{References}

1. Meier RK: Polycystic ovary syndrome. Nurs Clin North Am 53: 407-420, 2018.

2. Nandi A, Chen Z, Patel R and Poretsky L: Polycystic ovary syndrome. Endocrinol Metab Clin North Am 43: 123-147, 2014.

3. Goodarzi MO, Dumesic DA, Chazenbalk G and Azziz R: Polycystic ovary syndrome: Etiology, pathogenesis and diagnosis. Nat Rev Endocrinol 7: 219-231, 2011.

4. Ferriman D and Purdie AW: The aetiology of oligomenorrhoea and/or hirsuties: A study of 467 patients. Postgrad Med J 59: 17-20, 1983.

5. Balen AH, Conway GS, Kaltsas G, Techatrasak K, Manning PJ, West C and Jacobs HS: Polycystic ovary syndrome: The spectrum of the disorder in 1741 patients. Hum Reprod 10: 2107-2111, 1995

6. DeUgarte CM, Bartolucci AA and Azziz R: Prevalence of insulin resistance in the polycystic ovary syndrome using the homeostasis model assessment. Fertil Steril 83: 1454-1460, 2005.

7. Krentz AJ, von Muhlen D and Barrett-Connor E: Searching for polycystic ovary syndrome in postmenopausal women: Evidence of a dose-effect association with prevalent cardiovascular disease. Menopause 14: 284-292, 2007.

8. Jedel E, Waern M, Gustafson D, Landén M, Eriksson E, Holm G, Nilsson L, Lind AK, Janson PO and Stener-Victorin E: Anxiety and depression symptoms in women with polycystic ovary syndrome compared with controls matched for body mass index. Hum Reprod 25: 450-456, 2010.
9. Barthelmess EK and Naz RK: Polycystic ovary syndrome: Current status and future perspective. Front Biosci (Elite Ed) 6: 104-119, 2014.

10. Rothenberg SS, Beverley R, Barnard E, Baradaran-Shoraka M and Sanfilippo JS: Polycystic ovary syndrome in adolescents. Best Pract Res Clin Obstet Gynaecol 48: 103-114, 2018.

11. Nargis T and Chakrabarti P: Significance of circulatory DPP4 activity in metabolic diseases. IUBMB Life 70: 112-119, 2018.

12. Baumeier C, Schlüter L, Saussenthaler S, Laeger T, Rödiger M, Alaze SA, Fritsche L, Häring HU, Stefan N, Fritsche A, et al: Elevated hepatic DPP4 activity promotes insulin resistance and non-alcoholic fatty liver disease. Mol Metab 6: 1254-1263, 2017.

13. Kawasaki T, Chen W, Htwe YM, Tatsumi K and Dudek SM: DPP4 inhibition by sitagliptin attenuates LPS-induced lung injury in mice. Am J Physiol Lung Cell Mol Physiol 315: L834-1845, 2018.

14. Ozturk B, Gurbuz AS, Durak ZE and Ozturk HS: Dipeptidyl peptidase-4 and adenosine deaminase enzyme levels in polycystic ovary syndrome. Gynecol Endocrinol 35: 138-141, 2019.

15. Wawrzkiewicz-Jałowiecka A, Kowalczyk K, Trybek P, Jarosz T, Radosz P, Setlak M and Madej P: In search of New therapeutics-molecular aspects of the PCOS pathophysiology: Genetics, hormones, metabolism and beyond. Int J Mol Sci 21: 7054, 2020.

16. Yamagishi S, Nakamura N, Suematsu M, Kaseda K and Matsui T: Advanced glycation end products: A molecular target for vascular complications in diabetes. Mol Med 21 (Suppl 1): S32-S40, 2015.

17. Ishibashi Y, Matsui T, Maeda S, Higashimoto Y and Yamagishi S: Advanced glycation end products evoke endothelial cell damage by stimulating soluble dipeptidyl peptidase- 4 production and its interaction with mannose 6-phosphate/insulin-like growth factor II receptor. Cardiovasc Diabetol 12: 125, 2013.

18. Kaifu K, Ueda S, Nakamura N, Matsui T, Yamada-Obara N, Ando R, Kaida Y, Nakata M, Matsukuma-Toyonaga M, Higashimoto Y, et al: Advanced glycation end products evoke inflammatory reactions in proximal tubular cells via autocrine production of dipeptidyl peptidase-4. Microvasc Res 120: 90-93, 2018.

19. Diamanti-Kandarakis E, Alexandraki K, Piperi C, Aessopos A, Paterakis T, Katsikis I and Panidis D: Effect of metformin administration on plasma advanced glycation end product levels in women with polycystic ovary syndrome. Metabolism 56: 129-134, 2007

20. Merhi Z, Kandaraki EA and Diamanti-Kandarakis E: Implications and future perspectives of AGEs in PCOS pathophysiology. Trends Endocrinol Metab 30: 150-162, 2019.

21. Tahara N, Yamagishi S, Takeuchi M, Tahara A, Kaifu K, Ueda S, Okuda $S$ and Imaizumi T: Serum levels of advanced glycation end products (AGEs) are independently correlated with circulating levels of dipeptidyl peptidase-4 (DPP-4) in humans. Clin Biochem 46: 300-303, 2013.

22. Miki Y, Dambara H, Tachibana Y, Hirano K, Konishi M and Beppu M: Macrophage recognition of toxic advanced glycosylation end products through the macrophage surface-receptor nucleolin. Biol Pharm Bull 37: 588-596, 2014.

23. Kafali H, Iriadam M, Ozardali I and Demir N: Letrozole-induced polycystic ovaries in the rat: A new model for cystic ovarian disease. Arch Med Res 35: 103-108, 2004.

24. National Research Council: Subcommittee on Amphibian Standards: In: Amphibians: Guidelines for the breeding, care, and management of laboratory animals. National Academies Press, Washington, DC, 1974

25. Flecknell P: Replacement, reduction and refinement. Altex 19: 73-78, 2002.

26. Zhang S, Tu H, Yao J, Le J, Jiang Z, Tang Q, Zhang R, Huo P and Lei X: Combined use of Diane-35 and metformin improves the ovulation in the PCOS rat model possibly via regulating glycolysis pathway. Reprod Biol Endocrinol 18: 58, 2020.

27. Torres PJ, Skarra DV, Ho BS, Sau L, Anvar AR, Kelley ST and Thackray VG: Letrozole treatment of adult female mice results in a similar reproductive phenotype but distinct changes in metabolism and the gut microbiome compared to pubertal mice. BMC Microbiol 19: 57, 2019.

28. Venegas B, De León Gordillo LY, Rosas G, Espinoza JA, Morán C, Domínguez R and Morales-Ledesma L: In rats with estradiol valerate-induced polycystic ovary syndrome, the acute blockade of ovarian $\beta$-adrenoreceptors improve ovulation. Reprod Biol Endocrinol 17: 95, 2019.

29. Marcondes FK, Bianchi FJ and Tanno AP: Determination of the estrous cycle phases of rats: Some helpful considerations. Braz J Biol 62: 609-614, 2002 
30. He T, Sun Y, Zhang Y, Zhao S, Zheng Y, Hao G and Shi Y: MicroRNA-200b and microRNA-200c are up-regulated in PCOS granulosa cell and inhibit KGN cell proliferation via targeting PTEN. Reprod Biol Endocrinol 17: 68, 2019.

31. Nishi Y, Yanase T, Mu Y, Oba K, Ichino I, Saito M, Nomura M, Mukasa C, Okabe T, Goto K, et al: Establishment and characterization of a steroidogenic human granulosa-like tumor cell line, KGN, that expresses functional follicle-stimulating hormone receptor. Endocrinology 142: 437-445, 2001.

32. Yi S, Zheng B, Zhu Y, Cai Y, Sun H and Zhou J: Melatonin ameliorates excessive PINK1/Parkin-mediated mitophagy by enhancing SIRT1 expression in granulosa cells of PCOS. Am J Physiol Endocrinol Metab 319: E91-E101, 2020.

33. Geng X, Zhao J, Huang J, Li S, Chu W, Wang WS, Chen ZJ and Du Y: lnc-MAP3K13-7:1 inhibits ovarian GC proliferation in PCOS via DNMT1 downregulation-mediated CDKN1A promoter hypomethylation. Mol Ther 29: 1279-1293, 2021.

34. Livak KJ and Schmittgen TD: Analysis of relative gene expression data using real-time quantitative PCR and the 2(-Delta Delta C(T)) method. Methods 25: 402-408, 2001

35. Abouzeid AH and Torchilin VP: The role of cell cycle in the efficiency and activity of cancer nanomedicines. Expert Opin Drug Deliv 10: 775-786, 2013.

36. Nakanishi M: Regulation of cell cycle checkpoints in mammalian cells. Seikagaku 73: 343-350, 2001 (In Japanese).

37. Barnum KJ and O'Connell MJ: Cell cycle regulation by checkpoints. Methods Mol Biol 1170: 29-40, 2014.

38. Liu Q, Jiang J, Shi Y, Mo Z and Li M: Apelin/Apelin receptor: A new therapeutic target in polycystic ovary syndrome. Life Sci 260: 118310, 2020.

39. Khan MJ, Ullah A and Basit S: Genetic basis of polycystic ovary syndrome (PCOS): Current perspectives. Appl Clin Genet 12: 249-260, 2019.

40. Ajmal N, Khan SZ and Shaikh R: Polycystic ovary syndrome (PCOS) and genetic predisposition: A review article. Eur J Obstet Gynecol Reprod Biol X 3: 100060, 2019.

41. Abdalla M, Deshmukh H, Atkin SL and Sathyapalan T: miRNAs as a novel clinical biomarker and therapeutic targets in polycystic ovary syndrome (PCOS): A review. Life Sci 259: 118174, 2020.

42. Braga LDDC, Godoy-Matos AF, Siciliano PO, Corrêa JODA and Carvalho DP: Is DPP4 activity increased in PCOS? Diabetes Metab Syndr 12: 673-675, 2018.

43. Wang F, Zhang ZF, He YR, Wu HY and Wei SS: Effects of dipeptidyl peptidase-4 inhibitors on transforming growth factor-beta1 signal transduction pathways in the ovarian fibrosis of polycystic ovary syndrome rats. J Obstet Gynaecol Res 45: 600-608, 2019.

44. Kajiyama H, Kikkawa F, Maeda O, Suzuki T, Ino K and Mizutani S: Increased expression of dipeptidyl peptidase IV in human mesothelial cells by malignant ascites from ovarian carcinoma patients. Oncology 63: 158-165, 2002.

45. Barreira da Silva R, Laird ME, Yatim N, Fiette L, Ingersoll MA and Albert ML: Dipeptidylpeptidase 4 inhibition enhances lymphocyte trafficking, improving both naturally occurring tumor immunity and immunotherapy. Nat Immunol 16: 850-858, 2015.

46. Jensterle M, Goricar K and Janez A: Add on DPP-4 inhibitor alogliptin alone or in combination with pioglitazone improved $\beta$-cell function and insulin sensitivity in metformin treated PCOS. Endocr Res 42: 261-268, 2017.
47. Zhang Y, Hu M, Jia W, Liu G, Zhang J, Wang B, Li J, Cui P, $\mathrm{Li} X$, Lager S, et al: Hyperandrogenism and insulin resistance modulate gravid uterine and placental ferroptosis in PCOS-like rats. J Endocrinol 246: 247-263, 2020.

48. Rutkowska AZ and Diamanti-Kandarakis E: Do advanced glycation end products (AGEs) Contribute to the comorbidities of polycystic ovary syndrome (PCOS)? Curr Pharm Des 22: 5558-5571, 2016

49. Garg D and Merhi Z: Relationship between advanced glycation end products and steroidogenesis in PCOS. Reprod Biol Endocrinol 14: 71, 2016.

50. Diamanti-Kandarakis E, Katsikis I, Piperi C, Kandaraki E, Piouka A, Papavassiliou AG and Panidis D: Increased serum advanced glycation end-products is a distinct finding in lean women with polycystic ovary syndrome (PCOS). Clin Endocrinol (Oxf) 69: 634-641, 2008

51. Azhary JMK, Harada M, Kunitomi C, Kusamoto A, Takahashi N, Nose E, Oi N, Wada-Hiraike O, Urata Y, Hirata T, et al: Androgens increase accumulation of advanced glycation end products in granulosa cells by activating ER stress in PCOS. Endocrinology 161: bqaa015, 2020.

52. Pertynska-Marczewska M, Diamanti-Kandarakis E, Zhang J and Merhi Z: Advanced glycation end products: A link between metabolic and endothelial dysfunction in polycystic ovary syndrome? Metabolism 64: 1564-1573, 2015.

53. Diamanti-Kandarakis E, Piperi C, Patsouris E, Korkolopoulou P, Panidis D, Pawelczyk L, Papavassiliou AG and Duleba AJ: Immunohistochemical localization of advanced glycation end-products (AGEs) and their receptor (RAGE) in polycystic and normal ovaries. Histochem Cell Biol 127: 581-589, 2007.

54. Yamagishi S, Fukami K and Matsui T: Crosstalk between advanced glycation end products (AGEs)-receptor RAGE axis and dipeptidyl peptidase-4-incretin system in diabetic vascular complications. Cardiovasc Diabetol 14: 2, 2015.

55. Herman GA, Bergman A, Stevens C, Kotey P, Yi B, Zhao P, Dietrich B, Golor G, Schrodter A, Keymeulen B, et al: Effect of single oral doses of sitagliptin, a dipeptidyl peptidase- 4 inhibitor, on incretin and plasma glucose levels after an oral glucose tolerance test in patients with type 2 diabetes. J Clin Endocrinol Metab 91: 4612-4619, 2006.

56. Ferjan S, Janez A and Jensterle M: DPP4 inhibitor sitagliptin as a potential treatment option in metformin-intolerant obese Women with polycystic ovary syndrome: A pilot randomized study. Endocr Pract 24: 69-77, 2018.

57. Daneshjou D, Zadeh Modarres S, Soleimani Mehranjani M and Shariat Zadeh SMA: Comparing the effect of sitagliptin and metformin on the oocyte and embryo quality in classic PCOS patients undergoing ICSI. Ir J Med Sci 190: 685-692, 2021.

(7) $\mathrm{E}$ This work is licensed under a Creative Commons Attribution-NonCommercial-NoDerivatives 4.0 International (CC BY-NC-ND 4.0) License. 